\title{
Neutrophil-to-Lymphocyte Ratio Predicts Clinical Outcome of Severe Acute Exacerbation of COPD in Frequent Exacerbators
}

This article was published in the following Dove Press journal: International Journal of Chronic Obstructive Pulmonary Disease

\author{
Fang-Ying $\mathrm{Lu}^{1,2, *}$ \\ Rong Chen ${ }^{1,2, *}$ \\ Ning $\mathrm{Li}^{1,2}$ \\ Xian-Wen Sun ${ }^{1,2}$ \\ Min Zhou ${ }^{1,2}$ \\ Qing-Yun Li ${ }^{1,2}$ \\ Yi Guo ${ }^{1,2}$
}

\begin{abstract}
'Department of Respiratory and Critical Care Medicine, Ruijin Hospital, Shanghai Jiao Tong University School of Medicine, Shanghai, People's Republic of China; ${ }^{2}$ Institute of Respiratory Diseases, Shanghai Jiao Tong University School of Medicine, Shanghai, People's Republic of China
\end{abstract}

*These authors contributed equally to this work

Correspondence: Yi Guo

Department of Respiratory and Critical Care Medicine, Ruijin Hospital, Shanghai Jiao Tong University School of Medicine, No. 197, Rui jin 2nd Road, Shanghai, 200025, People's Republic of China

Fax +862164674301

Email guoyi62।@qq.com

Qing-Yun Li

Department of Respiratory and Critical Care Medicine, Ruijin Hospital, Shanghai Jiao Tong University School of Medicine, No. 197, Rui jin 2nd Road, Shanghai, 200025, People's Republic of China

Fax +86216467 430I

Email liqingyun68@hotmail.com
Purpose: Frequent exacerbators are a specific phenotype of chronic obstructive pulmonary disease (COPD), whose clinical characteristics and prognostic biomarkers during severe acute exacerbation (AECOPD) have not yet been fully elucidated. The aim of this study was to investigate the clinical features of severe AECOPD in frequent exacerbators and explore the predictive value of the neutrophil-to-lymphocyte ratio (NLR) for outcome in this phenotype during severe exacerbation.

Patients and Methods: A total of 604 patients with severe AECOPD were retrospectively included in the study. Subjects were defined as frequent exacerbators if they experienced two or more exacerbations in the past year. Clinical characteristics and worse outcome (ICU admission, or invasive ventilation, or in-hospital mortality) during severe AECOPD were compared between frequent exacerbators and non-frequent ones. Furthermore, the relationship between NLR and worse outcome in frequent exacerbators was analyzed using logistic regression and receiver operating characteristic (ROC).

Results: Among 604 patients with severe AECOPD, 282 (46.69\%) were frequent exacerbators and $322(53.31 \%)$ were non-frequent exacerbators. Compared with the non-frequent ones, frequent exacerbators presented higher levels of NLR (5.93 [IQR, 3.40-9.28] vs 4.41 [IQR, 2.74-6.80]; $p<0.001$ ), and more worse outcome incidence (58 [20.57\%] vs 38 [11.80\%]; $p=0.003)$. Moreover, among the frequent exacerbators, NLR levels in the patients with worse outcome were much higher than in those without worse outcome (11.09 [IQR, 7.74-16.49] vs 5.28 [IQR, 2.93-7.93]; $p<0.001)$. Increased NLR was significantly associated with a higher risk of worse outcome in frequent exacerbators (OR, 1.43; 95\% CI, 1.28-1.64; $p<0.001)$. Furthermore, ROC analysis revealed that a cut-off value of 10.23 , NLR could predict worse outcome of severe AECOPD in frequent exacerbators (sensitivity $62.1 \%$, specificity $92.0 \%$, AUC 0.833).

Conclusion: Frequent exacerbators exhibited an increased level of NLR and a higher proportion of worse outcome during severe AECOPD. NLR is expected to be a promising predictive biomarker for the prognosis of severe AECOPD in frequent exacerbators.

Keywords: COPD, frequent exacerbator, NLR, severe exacerbation, worse outcome

\section{Introduction}

Chronic obstructive pulmonary disease (COPD) is a global epidemic respiratory disease that influences about 175 million people worldwide and is estimated to become the fourth commonest cause of death by $2040 .^{1,2}$ In China, at least $13.7 \%$ of 40 years or older individuals suffer from this disease. ${ }^{3}$ On average, COPD patients generally experience 0.5 to 3.5 acute exacerbations of COPD (AECOPD) per year. ${ }^{4}$ 
According to Global Initiative for Chronic Obstructive Lung Disease (GOLD) 2020, exacerbation is defined as acute worsening of respiratory symptoms calling for additional therapy, where those cases that require hospitalization or visits to the emergency room are regarded as severe AECOPD. ${ }^{4}$ In the case of severe AECOPD, patients encounter serious inflammatory eruption and gas trapping, resulting in acute respiratory failure and even mortality risk. ${ }^{5-7}$ Patients with $\geq 2$ exacerbations experienced in the course of the past year are identified as 'frequent exacerbator phenotype ${ }^{, 4}$. Previous studies have reported that frequent exacerbators were associated with more serious airway obstruction, poorer health status and worse life quality. ${ }^{4,8-10}$ However, specific characteristics of frequent exacerbators with severe AECOPD remain unclear. In addition, limited research has focused on biomarkers predicting clinical outcome in these patients, especially those with severe exacerbation.

Neutrophils, the most abundant leukocytes in circulation, participate in multiple immune and inflammatory processes via phagocytosis, particle production, cytokine release and similar. ${ }^{11}$ The lymphocytes have been emphasized as the effectors and coordinators of inflammation in diverse pathophysiological mechanisms. ${ }^{12}$ The neutrophilto-lymphocyte ratio (NLR) in peripheral blood reflects the high level of neutrophils and the secondary development of lymphopenia in response to inflammatory stress. ${ }^{13}$ As a prognostic biomarker, NLR has received increasing attention in many inflammatory diseases such as pneumonia, pancreatitis, etc. ${ }^{13,14}$

COPD, especially severe AECOPD, was accompanied by excessive inflammatory response. ${ }^{15}$ The abnormal inflammation encompasses complex interactions of various immunocytes, including neutrophils and lymphocytes. ${ }^{15,16}$ Hence, NLR is expected to be a putative biomarker reflecting the disease severity and treatment effect in COPD. ${ }^{17,18}$ Nevertheless, little is known about the relationship between NLR and clinical outcome of severe AECOPD, especially for the frequent exacerbators. Thus, the current study aimed to investigate the clinical features and NLR's predictive value for outcome of severe AECOPD in frequent exacerbators.

\section{Patients and Methods}

\section{Subject Profile}

A total of 786 patients with severe AECOPD admitted to Ruijin Hospital between April 2016 and March 2020 were retrospectively enrolled in the current study. Patients who met the following criteria were included: a) COPD diagnosis at any of the previous clinical visits: with a postbronchodilator forced expiratory volume in 1 second $\left(\mathrm{FEV}_{1}\right)$ /forced vital capacity $(\mathrm{FVC}$ ) value $<0.7$ in the stable stage; b) primary diagnosis of severe AECOPD at this admission: acute worsening of respiratory symptom that requires hospitalization. ${ }^{4}$ Patients complicated with other respiratory diseases were excluded, such as asthma, lung cancer, bronchiectasis, interstitial lung diseases, etc. In addition, patients with antibiotic or corticosteroid treatment within one month prior to enrollment were not listed in the study. Finally, 604 subjects were included in the analysis (Figure 1). All patients provided written informed consent for scientific research of clinical data during hospitalization.

This study was conducted in accordance with the Declaration of Helsinki, and the protocol was reviewed and approved by the institutional review board of Ruijin Hospital.

\section{Data and Sample Collection}

Based on the definition of 'frequent exacerbation' by GOLD 2020, ${ }^{4}$ the patients were divided into frequent exacerbators (subjects who experienced two or more exacerbations within the past year) and non-frequent ones. We collected demographics and clinical characteristics from the electronic medical record, including the spirometry parameters in stable stage within the last two years. Blood cell count and other inflammatory indicators were obtained on the day of admission before the administration of antibiotics and steroids. Furthermore, the NLR value (count of neutrophil/count of lymphocyte) of each subject was calculated and analyzed. We recorded the clinical outcome of each participant. Patients meeting any of the three criteria were defined as having worse outcome: needing invasive ventilation, intensive care unit (ICU) admission, or in-hospital mortality. ${ }^{19}$

\section{Statistical Analysis}

Continuous variables were expressed as mean with standard deviation (SD) for normally distributed variables or median with interquartile range (IQR) for skewed variables. Quantity and percentage were employed for categorical variables. Differences in clinical characters between the two groups were evaluated using $t$-test and MannWhitney U-test for continuous variables, while the Chisquared test and Fisher exact probability method were 


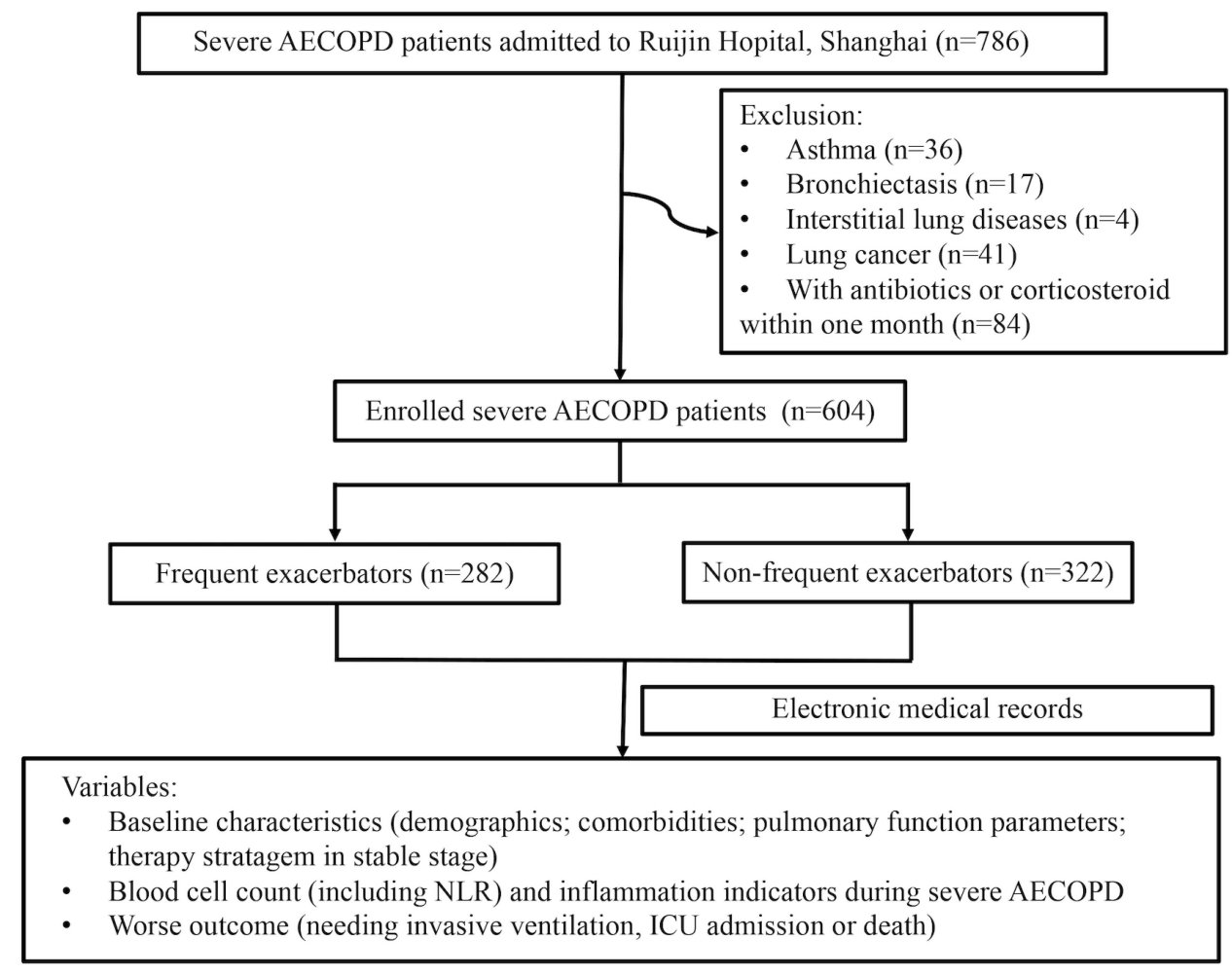

Figure I Flowchart of the study participants.

Abbreviations: AECOPD, acute exacerbation of chronic obstructive pulmonary disease; NLR, neutrophil-to-lymphocyte ratio; ICU, intensive care unit.

used for categorical variables, as appropriate. Spearman correlation coefficient (r) was calculated to identify the existence of correlation. In addition, non-parameter imputation method, missForest procedure in $\mathrm{R}$, was also applied to estimate the missing indices, ${ }^{20}$ which were further used for logistic regression analysis.

Moreover, we conducted multivariate logistic regression models to assess the association between NLR and worse outcome in frequent exacerbators. With the first quartile of NLR as a reference, Odds ratios (OR) and $95 \%$ confidence intervals $(95 \% \mathrm{CI})$ for the second to fourth quartiles were calculated, respectively. Meanwhile, ORs for the incident of worse outcome were also tested for per one unit increase when NLR was considered as original continuous variables. In addition to the crude model, model 1 was age-, sex-, body mass index (BMI)- and smoking status adjusted model and model 2 was further adjusted for comorbidities. Model 3 was further adjusted for GOLD grade and maintenance therapy of COPD. In model 4, inflammatory indicators were further adjusted based on model 3. The covariates, which were known or considered to be related to NLR and prognosis, were selected and adjusted with a stepwise method.
Furthermore, receiver operating characteristic (ROC) curve analysis was employed to evaluate the sensitivity and specificity of NLR in predicting worse outcome, as well as its cut-off value. All statistical analyses were performed on $\mathrm{R}$ version 4.0.0, and a two-tailed $p$-value less than 0.05 was considered as statistically significant.

\section{Results}

\section{Clinical Characteristics of Frequent and Non-Frequent Exacerbators During Severe AECOPD}

Among the 604 patients with severe AECOPD (male/ female 523/81; median age 77 [IQR, 67-84]), 282 patients $(46.69 \%)$ were frequent exacerbators, and 322 patients (53.31\%) were non-frequent ones. Clinical characteristics of participants are listed in Table 1 . There were no significant differences in age, sex, BMI and smoking status between the two groups. Among the 279 patients who underwent pulmonary function test, frequent exacerbators $(n=140)$ showed more deteriorated airflow limitation than non-frequent ones $(\mathrm{n}=139)$, presented with lower level of $\mathrm{FEV}_{1} \%$ predicted $\left(\mathrm{FEV}_{1} \%\right.$ pred; 36.25 [IQR, 26.7849.69] vs 42.91 [IQR, 27.80-57.03] \%; $p=0.004)$, 
Table I Characteristics of Frequent Exacerbators and Non-Frequent Exacerbators During Severe AECOPD

\begin{tabular}{|c|c|c|c|c|}
\hline Characteristics & Total $(n=604)$ & Frequent Exacerbators $(n=282)$ & Non-Frequent Exacerbators $(n=322)$ & $P$ value \\
\hline Age, years & $77.00(67.00-84.00)$ & $78.00(68.00-84.00)$ & $76.00(67.00-84.00)$ & 0.127 \\
\hline Male, n (\%) & $523(86.59)$ & 247 (87.59) & $276(85.7 I)$ & 0.500 \\
\hline BMI, kg/m² & $21.91(19.02-24.75)$ & $21.54(18.60-24.78)$ & $22.03(19.36-24.72)$ & 0.630 \\
\hline Current smoker, n (\%) & $144(23.84)$ & $58(20.57)$ & $86(26.7 I)$ & 0.077 \\
\hline \multicolumn{5}{|l|}{ Comorbidities, n (\%) } \\
\hline Diabetes & $120(19.87)$ & $56(19.86)$ & $64(19.88)$ & 0.996 \\
\hline Hypertension & $321(53.15)$ & $160(56.74)$ & $16 \mid(50.00)$ & 0.098 \\
\hline NYHA $\geq 3$ & $95(15.73)$ & $47(16.66)$ & $48(|4.9|)$ & 0.553 \\
\hline Arrhythmia & $130(2 \mid .52)$ & $72(25.53)$ & $58(18.01)$ & $0.025^{*}$ \\
\hline \multicolumn{5}{|l|}{ Pulmonary Function Test ${ }^{\mathrm{a}}$} \\
\hline $\mathrm{FEV}_{1} / \mathrm{FVC}, \%$ & $52.08(42.25-66.67)$ & $49.09(37.80-56.75)$ & $60.24(43.50-72.73)$ & $0.007^{*}$ \\
\hline FEV , pred, \% & $37.50(27.25-53.78)$ & $36.25(26.78-49.69)$ & $42.91(27.80-57.03)$ & $0.004^{*}$ \\
\hline FVC pred, \% & $57.40 \pm 17.50$ & $55.22 \pm 16.18$ & $59.57 \pm 18.52$ & $0.038^{*}$ \\
\hline GOLD grade, $\mathrm{I} / \mathrm{II} / \mathrm{III} / \mathrm{I} \mathrm{V}^{\mathrm{b}}$ & $|8 / 68 /| 102 / 9 \mid$ & $7 / 27 / 55 / 51$ & $\mid \mathrm{I} / 4 \mathrm{I} / 47 / 40$ & $0.030 *$ \\
\hline \multicolumn{5}{|c|}{ Therapy in stable stage, $\mathrm{n}(\%)$} \\
\hline LABA monotherapy & $8(1.32)$ & $6(2.13)$ & $2(0.62)$ & 0.100 \\
\hline LAMA monotherapy & $10(1.66)$ & $2(0.7 I)$ & $8(2.48)$ & 0.076 \\
\hline$L A B A+I C S$ & $87(14.40)$ & $45(I 5.96)$ & $42(13.04)$ & 0.309 \\
\hline LAMA+LABA & $2(0.33)$ & $\mathrm{I}(0.35)$ & $\mathrm{I}(0.3 \mathrm{I})$ & 1.000 \\
\hline$L A B A+L A M A+I C S$ & $160(24.69)$ & $108(38.30)$ & $52(16.15)$ & $<0.001 *$ \\
\hline \multicolumn{5}{|l|}{ Blood cell count } \\
\hline Leukocytes, $\times 10^{9} / \mathrm{L}$ & $7.71(5.95-10.25)$ & $8.25(6.15-10.84)$ & $7.36(5.80-9.83)$ & $0.008^{*}$ \\
\hline Neutrophils, $\times 10^{9} / \mathrm{L}$ & $5.75(3.87-8.18)$ & $6.14(4.20-8.82)$ & $5.26(3.73-7.69)$ & $<0.001 *$ \\
\hline Lymphocytes, $\times 10^{9} / \mathrm{L}$ & $1.15(0.84-1.59)$ & $1.08(0.78-1.48)$ & $1.23(0.93-1.63)$ & $0.002 *$ \\
\hline NLR & $5.05(2.92-8.16)$ & $5.93(3.40-9.28)$ & $4.41(2.74-6.80)$ & $<0.001 *$ \\
\hline Monocytes, $\times 10^{9} / \mathrm{L}$ & $0.53(0.36-0.73)$ & $0.55(0.32-0.72)$ & $0.52(0.38-0.75)$ & 0.348 \\
\hline Eosinophils, $\times 10^{9} / \mathrm{L}$ & $0.07(0.01-0.19)$ & $0.04(0.00-0.17)$ & $0.10(0.01-0.21)$ & $<0.001 *$ \\
\hline Haemoglobin, g/L & $128.00(|| 5.00-14 \mid .00)$ & $127.00(\mid 16.00-139.75)$ & $\mid 29.00(|| 4.00-\mid 42.00)$ & 0.931 \\
\hline Platelet, $\times 10^{9} / \mathrm{L}$ & $189.00(151.00-245.00)$ & $188.00(151.25-243.00)$ & $191.50(151.00-245.00)$ & 0.726 \\
\hline \multicolumn{5}{|l|}{ Inflammatory parameters } \\
\hline CRP, $\mathrm{mg} / \mathrm{L}^{\mathrm{c}}$ & $13.00(4.00-48.40)$ & II.75 (3.73-46.43) & $14.00(4.15-52.50)$ & 0.351 \\
\hline $\mathrm{PCT}, \mathrm{ng} / \mathrm{mL}^{\mathrm{d}}$ & $0.05(0.05-0.14)$ & $0.05(0.05-0.14)$ & $0.05(0.05-0.14)$ & 0.800 \\
\hline $\mathrm{ESR}, \mathrm{mm} / \mathrm{h}^{\mathrm{e}}$ & $14.00(6.00-36.00)$ & $13.50(6.00-34.75)$ & $14.00(7.00-36.00)$ & 0.300 \\
\hline$L D H, I U / L^{f}$ & $179.00(|43.00-2| 2.00)$ & $183.00(\mid 45.25-225.25)$ & $171.00(|4| .25-201.00)$ & $0.016 *$ \\
\hline Worse outcome (\%) & $96(15.89)$ & $58(20.57)$ & $38(11.80)$ & $0.003^{*}$ \\
\hline ICU admission (\%) & $77(\mid 2.75)$ & $47(16.67)$ & $30(9.32)$ & $0.007^{*}$ \\
\hline Invasive ventilation (\%) & $15(2.48)$ & II (3.90) & $4(1.24)$ & $0.036^{*}$ \\
\hline Mortality (\%) & $32(5.30)$ & $23(8.16)$ & $9(2.80)$ & $0.003^{*}$ \\
\hline
\end{tabular}

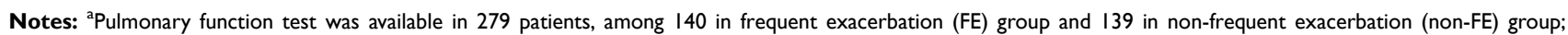
${ }^{b}$ GOLD grade was classified according to pulmonary function test; ${ }^{\mathrm{C} C R P}$ was available in 535 patients, among 248 in $\mathrm{FE}$ group and 287 in non-FE group; ${ }^{\mathrm{d}} \mathrm{PCT}$ was available in 468 patients, among 225 in FE group and 243 in non-FE group; ${ }^{e}$ ESR was available in 405 patients, among 186 in FE group and 219 in non-FE group; ${ }^{\mathrm{L}} \mathrm{LDH}$ was available in 512 patients, among 250 in FE group and 262 in non-FE group, ${ }^{*} p<0.05$.

Abbreviations: BMI, body mass index; NYHA, New York heart association; FEV , forced expiratory volume in Is; FVC, forced vital capacity; LABA, long-acting betaagonists; LAMA, long-acting antimuscarinic antagonists; ICS, inhaled corticosteroids; NLR, neutrophil to lymphocyte ratio; CRP, C-reactive protein; PCT, procalcitonin; ESR, erythrocyte sedimentation rate; $L D H$, lactic dehydrogenase; ICU, intensive care unit. 

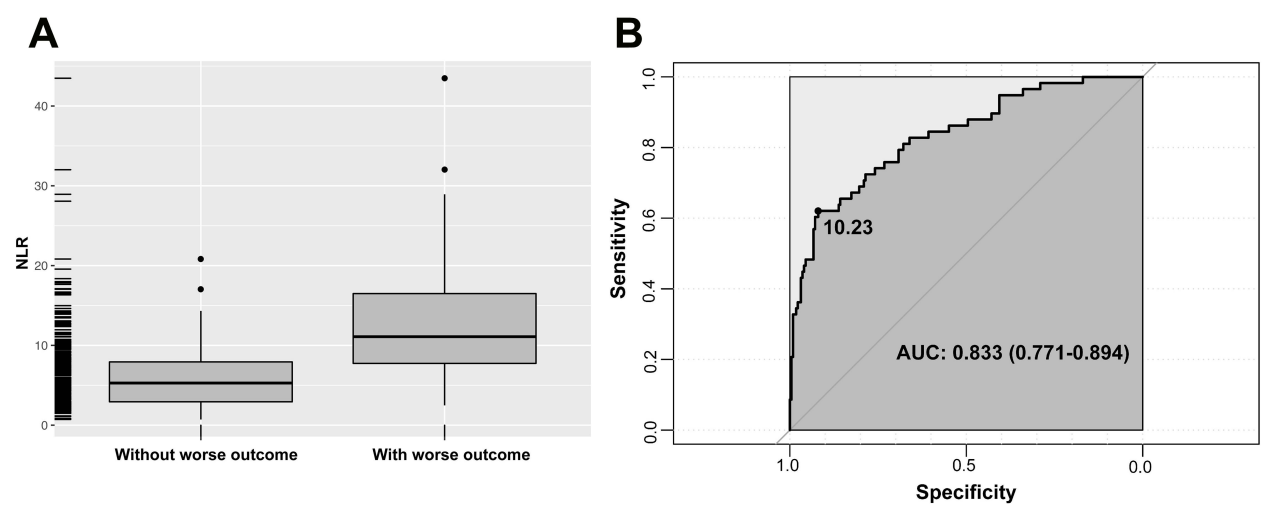

Figure 2 (A) NLR during severe AECOPD in frequent exacerbators with different outcome. Among the frequent exacerbators, the values of NLR in the ones with worse outcome were higher than in those without worse outcomes. (B) ROC curve of NLR for predicting the worse outcome of severe AECOPD in frequent exacerbators. The cut-off value of NLR was 10.23, with a sensitivity of 0.62 I, a specificity of 0.920 and an AUC of $0.833(95 \% \mathrm{Cl} 0.77 \mathrm{I}-0.894 ; p<0.00 \mathrm{I})$.

Abbreviations: NLR, neutrophil-to-lymphocyte ratio; AECOPD, acute exacerbation of chronic obstructive pulmonary disease; ROC, receiver operating characteristic; AUC, area under the curve.

decreased FVC percent predicted (FVC\% pred; 55.22 \pm 16.18 vs $59.57 \pm 18.52 \% ; p=0.038)$ and lower $\mathrm{FEV}_{1} / \mathrm{FVC}$ post-bronchodilator (49.09 [IQR, 37.80-56.75] vs 60.24 [IQR, 43.50-72.73] \%; $p=0.007)$, as well as worse GOLD grade $(p=0.030)$. Frequent exacerbators had a higher incidence of arrhythmia than the non-frequent ones (72 [25.53\%] vs 58 [18.01\%]; $p=0.025)$.

As for therapeutic intervention in the stable stage, frequent exacerbators were also more likely to undergo triple inhaled therapy (long-acting antimuscarinic antagonists +long-acting beta-agonists +inhaled corticosteroids) than non-frequent ones (108 [38.30\%] vs 52 [16.15\%]; $p<0.001)$.

\section{NLR Levels of Frequent and Non-Frequent Exacerbators During Severe AECOPD}

Regarding laboratory findings during severe AECOPD (Table 1), frequent exacerbators exhibited increased count of leukocytes (8.25 [IQR, 6.15-10.84] vs 7.36 [IQR, 5.80-$\left.9.83] \times 10^{9} / \mathrm{L} ; p=0.008 ;\right)$ and neutrophils $(6.14$ [IQR, 4.20-$8.82]$ vs $\left.5.26 \quad[\mathrm{IQR}, 3.73-7.69] \times 10^{9} / \mathrm{L} ; \quad p<0.001\right)$. Meanwhile, decreased count of lymphocytes (1.08 [IQR, $0.78-1.48]$ vs 1.23 [IQR, 0.93-1.63] $\left.\times 10^{9} / \mathrm{L} ; p=0.002\right)$ and eosinophils (0.04 [IQR, 0.00-0.17] vs 0.10 [IQR, 0.01-0.21] $\times 10^{9} / \mathrm{L} ; p<0.001$ ) were found in the frequent exacerbators. Marked elevation of NLR was observed in frequent exacerbators compared with the non-frequent ones (5.93 [IQR, 3.40-9.28] vs 4.41 [IQR, 2.74-6.80]; $p<0.001$ ). In addition, frequent exacerbation group also showed higher level of lactic dehydrogenase (LDH; 183.00 [IQR, 145.25-225.25] vs 171.00 [IQR, 141.25-201.00]; $p=0.016$ ).

\section{The Relationship Between NLR Values and Worse Outcome of Severe AECOPD in Frequent Exacerbators}

A total of $96(15.89 \%)$ patients exhibited the worse outcome, including 15 (2.48\%) who received invasive ventilation, 77 $(12.75 \%)$ who were admitted to ICU, and 33 (5.30\%) who deceased in hospital. Worse outcome was more commonly observed in frequent exacerbators than in non-frequent ones (58 [20.57\%] vs 38 [11.80\%]; $p=0.003$; Table 1). Moreover, we further divided the frequent exacerbators into two subgroups, with or without worse outcome; and we found that NLR was higher in the subjects with worse outcome than those without worse outcome (11.09 [IQR, 7.74-16.49] vs 5.28 [IQR, 2.93-7.93]; $p<0.001$; Figure 2A). In addition, a higher proportion of female patients, more complications with hypertension and New York heart association (NYHA) $\geq 3$, fewer count of eosinophils, lower level of hemoglobin, a higher level of procalcitonin (PCT) and LDH, and lower proportion of triple inhaled therapy in stable stage $(p<0.05$ for all) were also observed in patients with worse outcome (Table 2).

\section{NLR Predicted the Worse Outcome of Severe AECOPD in Frequent Exacerbators}

Considering the higher level of NLR in the frequent exacerbators with worse outcome, we extended the observation between NLR and clinical prognosis. We found that the value of NLR was positively correlated with the occurrence of worse outcome $(\mathrm{r}=0.466, p<0.001)$. Multivariable-adjusted logistic regression models revealed that increased NLR was associated 
Table 2 Characteristics of Frequent Exacerbators with Different Outcome During Severe AECOPD

\begin{tabular}{|c|c|c|c|}
\hline & With Worse Outcome $(n=58)$ & Without Worse Outcome $(n=224)$ & $P$ value \\
\hline Age, years & $80.00(71.25-84.75)$ & $77.00(67.00-84.00)$ & 0.169 \\
\hline Male, n (\%) & $46(79.31)$ & $201(89.73)$ & $0.032 *$ \\
\hline BMI, $\mathrm{kg} / \mathrm{m}^{2}$ & $21.91 \pm 5.00$ & $22.03 \pm 4.19$ & 0.081 \\
\hline Current smoker, n (\%) & $7(12.07)$ & $51(22.77)$ & 0.072 \\
\hline Diabetes, n (\%) & $16(27.59)$ & $40(17.86)$ & 0.098 \\
\hline Hypertension, n (\%) & $42(72.4 \mathrm{I})$ & $118(52.68)$ & $0.007^{*}$ \\
\hline NYHA $\geq 3, n(\%)$ & $21(36.2 I)$ & $26(11.6 I)$ & $<0.001 *$ \\
\hline Arrhythmia, n (\%) & $16(27.59)$ & $56(25.00)$ & 0.687 \\
\hline GOLD grade, $\mathrm{I} / \mathrm{II} / \mathrm{II} / \mathrm{IV} \mathrm{V}^{\mathrm{a}}$ & $0 / 2 / 4 / 4$ & $7 / 25 / 51 / 47$ & 0.901 \\
\hline ICS+LABA+LAMA, n (\%) & $10(17.24)$ & $98(43.75)$ & $<0.00 I^{*}$ \\
\hline NLR & II.09 (7.74-16.49) & $5.28(2.93-7.93)$ & $<0.001 *$ \\
\hline Monocytes, $\times 10^{9} / \mathrm{L}$ & $0.49(0.23-0.66)$ & $0.55(0.34-0.73)$ & 0.065 \\
\hline Eosinophils, $\times 10^{9} / \mathrm{L}$ & $0.01(0.00-0.05)$ & $0.06(0.00-0.18)$ & $<0.00 I^{*}$ \\
\hline Haemoglobin, g/L & $122.00(102.00-133.00)$ & $129.00(\mid 18.00-141.00)$ & $0.002^{*}$ \\
\hline Platelet, $\times 10^{9} / \mathrm{L}$ & $179.50(\mid 42.75-220.00)$ & $191.00(152.00-248.00)$ & 0.102 \\
\hline CRP, mg/L ${ }^{b}$ & $15.35(5.33-72.08)$ & $11.00(3.10-42.75)$ & 0.069 \\
\hline $\mathrm{PCT}, \mathrm{ng} / \mathrm{mL}^{\mathrm{c}}$ & $0.09(0.05-0.55)$ & $0.05(0.05-0.10)$ & $<0.001 *$ \\
\hline $\mathrm{ESR}, \mathrm{mm} / \mathrm{h}^{\mathrm{d}}$ & $10.00(4.00-24.50)$ & $15.00(6.00-37.00)$ & 0.172 \\
\hline $\mathrm{LDH}, \mathrm{IU} / \mathrm{L}^{\mathrm{e}}$ & $207.50(176.75-266.25)$ & $179.50(|42.25-2| 3.75)$ & $<0.001 *$ \\
\hline
\end{tabular}

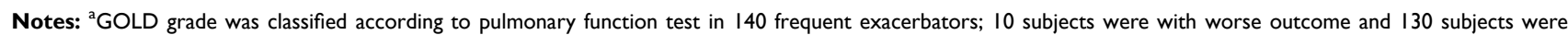
without worse outcome; ${ }^{b} \mathrm{CRP}$ was available for 248 frequent exacerbators, among whom 58 were the ones with worse outcome and 190 were without worse outcome; 'PCT was available for 225 frequent exacerbators, where 52 were with worse outcome and 173 were without worse outcome; ${ }^{d}$ ESR was available for I86 frequent exacerbators, where 43 were with worse outcome and I 43 were without worse outcome; ${ }^{\text {LDH }}$ was available for 250 frequent exacerbators, among whom 56 were with worse outcome and 194 were without worse outcome. ${ }^{*} p<0.05$.

Abbreviations: BMI, body mass index; NYHA, New York heart association; LABA, long-acting beta-agonists; LAMA, long-acting antimuscarinic antagonists; ICS, inhaled corticosteroids; NLR, neutrophil to lymphocyte ratio; CRP, C-reactive protein; PCT, procalcitonin; ESR, erythrocyte sedimentation rate; LDH, lactic dehydrogenase.

with a higher risk of worse outcome (Table 3 ). In the crude model, comparing with the first quartile of NLR, the OR of worse outcome for the third and fourth quartiles of NLR was 6.43 (95\% CI, 1.64-42.65; $p=0.018)$ and 39.73 (95\% CI, 11.24-253.52; $p<0.001)$, respectively. After adjusting for age, sex, BMI and smoking status, the adjusted OR were 6.51 (95\% CI, 1.63-43.71; $p=0.019$ ) and 42.87 (95\% CI, 11.74-279.01; $p<0.001)$. Following the further adjustment for comorbidities, GOLD grade and triple inhaled therapy, the association remained significant (all $p<0.05$ ). When the model was further adjusted for inflammatory indicators, a significantly higher risk of worse outcome was found in the fourth quartiles of NLR subgroup, with an OR of 41.85 (95\% CI, 9.57-306.74; $p<0.001)$. Meanwhile, in this multivariable-adjusted model, the OR of worse outcome in NLR was 1.43 (95\% CI, 1.28$1.64 ; p<0.001)$.

Finally, we performed a ROC curve analysis to evaluate the efficiency of NLR in predicting the worse outcome of severe AECOPD in frequent exacerbators (Figure 2B). With an NLR cut-off value of 10.23 , the sensitivity and specificity for the worse outcome were $62.1 \%$ and $92.0 \%$, respectively (area under the curve 0.833 ; 95\% CI $0.771-$ $0.894 ; p<0.001)$.

\section{Discussion}

In the present study, we found that frequent exacerbators showed an increased level of NLR and a higher proportion of worse outcome during severe AECOPD than non-frequent ones. Multivariable-adjusted logistic regression models further revealed that increased NLR was associated with a higher risk of worse outcome in frequent exacerbators. Moreover, NLR was a promising indicator for predicting the worse outcome of severe AECOPD in frequent exacerbators.

COPD is a heterogeneous disease. Identifying clinical phenotypes and conducting individual therapy stratagem should benefit COPD patients. $^{21}$ The landmark ECLIPSE study emphasized a unique subgroup of COPD, in which individuals with at least two exacerbations per year were classified as frequent exacerbators. ${ }^{9}$ Host-specific factors related to frequent exacerbation included the exacerbation history, complex comorbidities, deteriorated lung function, persistent airway inflammation, etc. ${ }^{8-10}$ In addition, Lipson et al, ${ }^{22}$ proved that frequent exacerbators were more frequently suggested triple therapies, especially in the prevention of severe AECOPD and the reduction of poor prognosis. Similarly, up to $46 \%$ of the subjects in our study were frequent exacerbators, accompanied 
Table 3 Association Between NLR and Worse Outcome in Frequent Exacerbators During Severe AECOPD

\begin{tabular}{|c|c|c|c|c|c|}
\hline & & \multicolumn{4}{|c|}{ NLR Quartiles } \\
\hline & NLR (per unit) & QI & Q2 & Q3 & Q4 \\
\hline Median of NLR & & 2.40 & 4.75 & 7.42 & 12.37 \\
\hline Interquartile range of NLR & & $1.90-2.84$ & $4.02-5.34$ & $6.72-8.38$ & $10.28-16.35$ \\
\hline adverse outcome, n (\%) & & $2(2.82)$ & $7(10.00)$ & II (I5.7I) & $38(53.52)$ \\
\hline \multicolumn{6}{|c|}{ OR $(95 \% \mathrm{Cl})$ for adverse outcome } \\
\hline Crude model & $1.37(1.26-1.50)^{* * *}$ & 1.00 (Ref) & $3.83(0.89-26.38)$ & $6.43(1.64-42.65)^{*}$ & $39.73(11.24-253.52)^{* * *}$ \\
\hline Model I $\mathrm{I}^{\mathrm{a}}$ & $1.39(1.27-1.54)^{* * *}$ & I.00 (Ref) & $4.01(0.91-27.90)$ & $6.5 \mathrm{I}(1.63-43.71)^{*}$ & $42.87(\mathrm{II} .74-279.01)^{* * *}$ \\
\hline Model $2^{b}$ & $1.43(1.29-1.60)^{* * *}$ & 1.00 (Ref) & $3.83(0.78-28.75)$ & $5.94(1.38-42.11)^{*}$ & $56.25(|3.86-40| .90)^{* * *}$ \\
\hline Model $3^{c}$ & $1.43(1.28-1.63)^{* * *}$ & I.00 (Ref) & $3.96(0.79-30.02)$ & $4.76(1.10-33.52)^{*}$ & $53.19(12.39-387.45)^{* * *}$ \\
\hline Model $4^{\mathrm{d}}$ & $1.43(1.28-1.64)^{* * *}$ & 1.00 (Ref) & $4.10(0.80-31.36)$ & $3.79(0.82-27.46)$ & $41.85(9.57-306.74)^{* * *}$ \\
\hline
\end{tabular}

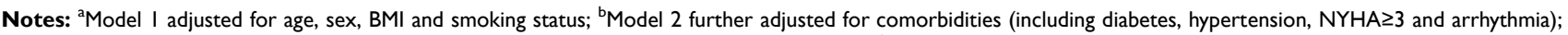
'Model 3 further adjusted for GOLD grade and triple inhaled therapy as maintenance strategy; ${ }^{\mathrm{d}}$ Model 4 further adjusted for inflammatory indicators (including CRP, PCT, ESR, LDH), $* p<0.05 ; * * * p<0.001$.

Abbreviations: NLR, neutrophil to lymphocyte ratio; AECOPD, acute exacerbation of chronic obstructive pulmonary disease; OR, Odd ratios; Cl, confidence intervals; BMI, body mass index; NYHA, New York heart association; CRP, C-reactive protein; PCT, procalcitonin; ESR, erythrocyte sedimentation rate; LDH, lactic dehydrogenase.

by worse GOLD grade and a higher proportion of arrhythmia and triple therapies.

Despite limited data emphasizing the AECOPD in frequent exacerbators, Matkovic et al, ${ }^{23}$ revealed that previous exacerbation history increased the risk of poor outcome during AECOPD. As most exacerbations tend to be triggered by infection, we took the assessment measure of other respiratory infectious diseases as a reference. ${ }^{19}$ From current research, worse outcome, including needing invasive ventilation, or ICU admission, or death in hospital, was employed to comprehensively evaluate the short-term prognosis of severe AECOPD. Consistent with prior studies, ${ }^{23}$ we also observed that more frequent exacerbators suffered from the worse outcome. Therefore, early identification, which requires effective indicators or biomarkers, is essential.

Our results revealed that the NLR value in frequent exacerbators was much higher than that in non-frequent ones during severe AECOPD. As previous studies reported, frequent exacerbation might be associated with dysbiosis in lower airway flora and impaired antiviral immunity. $^{24,25}$ Infection further increased, thus overactivating the neutrophils in the local and systemic responses. Another research also demonstrated that frequent exacerbators were more suitable for the treatment directed towards neutrophilic inflammation. ${ }^{24}$ These findings indicated frequent exacerbation phenotype might be complicated with more serious neutrophil inflammation. On the other hand, a recent report by Geerdink et al, ${ }^{26}$ have demonstrated that frequent exacerbators were accompanied by decreased lymphocytes, including CD8+ $\mathrm{T}$ effector memory cells and CD4+ $\mathrm{T}$ central memory cells. Notably, Lee et al, ${ }^{17}$ also confirmed that a higher level of NLR in AECOPD was associated with future exacerbations during the first-year follow-up period.

Moreover, we found that frequent exacerbators with worse outcome presented much higher value of NLR during severe AECOPD, and higher NLR indicated the risk of worse outcome. Importantly, Xiong et al, ${ }^{27}$ verified the similar association between NLR and poor prognosis in AECOPD, while our study focused on the outcome of severe AECOPD in frequent exacerbators. We demonstrated that NLR $>10.23$ was a potential prognostic biomarker for worse outcome of severe AECOPD. It was comparable to the research of Yao et al, ${ }^{28}$ who validated NLR $>6.24$ as the predictor of in-hospital mortality in AECOPD patients. It has been found that NLR could predict the need for invasive ventilation with the cut-off of $10.345,{ }^{29}$ while NLR $>16$ was identified as an independent mortality risk factor for AECOPD patients requiring ICU admission. $^{30}$ The different cut-off values of NLR in these studies may due to the different study designs and observational end-point. Our results may imply that speculated highlevel systematic inflammation and serious disease severity of exacerbation accounted for frequent exacerbator phenotype.

In addition to the value of NLR, which was different in frequent and non-frequent exacerbators, we also found another specific phenomenon during severe AECOPD, i.e. frequent 
exacerbators had fewer counts of eosinophils, especially the ones with worse outcome. Although previous studies suggested that frequent exacerbators presented eosinophilia, the samples were collected during the stable stage in most of these studies. $^{31,32}$ However, it was uncertain whether the dominant role of eosinophil remained during exacerbation. Also, $\mathrm{Wu}$ et al have demonstrated that the lower eosinophilic state was associated with poor prognosis, ${ }^{33}$ which was consistent with our findings. Therefore, the association between frequent exacerbations and eosinophils during severe AECOPD is worth further exploration.

There are some limitations in the present study. First, due to the retrospective nature of the study, some clinical information, such as details on smoking status, pulmonary function parameters and inflammatory indicators, were only available for some of the participants. Second, a single-center study may lead to data bias, thus, the reported results need to be further verified by a larger multi-center study. Finally, prospective studies are necessary to draw a more definite conclusion.

\section{Conclusion}

In conclusion, the present study reported an increased value of NLR and a higher proportion of worse outcome in frequent exacerbators during severe AECOPD. As a simple and cost-effective index, NLR is expected to be a promising biomarker to predict the clinical outcome of severe AECOPD in frequent exacerbators.

\section{Acknowledgments}

Thanks for the support from Innovative research team of high-level local universities in Shanghai.

\section{Author Contributions}

All authors made substantial contributions to conception and design, acquisition of data, or analysis and interpretation of data; took part in drafting the article or revising it critically for important intellectual content; agreed to submit to the current journal; gave final approval of the version to be published; and agree to be accountable for all aspects of the work.

\section{Funding}

This work was supported by National Key R\&D Program of China [granted number 2017YFC1309701, 2017YFC1309700 and 2018YFC1311900]; National Natural Science Foundation of China [granted number 81570029]; Grant of Beijing Bethune Charitable Foundation [granted number BJ-
RW2020003J] and Shanghai Key Discipline for Respiratory Diseases [granted number 2017ZZ02014].

\section{Disclosure}

The authors report no conflicts of interest in this work.

\section{References}

1. Soriano JB, Abajobir AA, Abate KH, Collaborators GBDCRD. Global, regional, and national deaths, prevalence, disability-adjusted life years, and years lived with disability for chronic obstructive pulmonary disease and asthma, 1990-2015: a systematic analysis for the global burden of disease study 2015. Lancet Respir Med. 2017;5(9):691-706. doi:10.1016/S2213-2600(17)30293-X

2. Foreman KJ, Marquez N, Dolgert A, et al. Forecasting life expectancy, years of life lost, and all-cause and cause-specific mortality for 250 causes of death: reference and alternative scenarios for 2016-40 for 195 countries and territories. Lancet. 2018;392 (10159):2052-2090. doi:10.1016/S0140-6736(18)31694-5

3. Wang C, Xu J, Yang L, et al. Prevalence and risk factors of chronic obstructive pulmonary disease in China (the China pulmonary health [CPH] study): a national cross-sectional study. Lancet. 2018;391 (10131):1706-1717. doi:10.1016/S0140-6736(18)30841-9

4. Global Initiative for Chronic Obstructive Lung Disease [home page on Internet]. Global strategy for diagnosis, management, and prevention of COPD (updated 2020). Available from: http://www.goldcopd. org. Accessed October 16, 2020.

5. Dransfield MT, Kunisaki KM, Strand MJ, et al. Acute exacerbations and lung function loss in smokers with and without chronic obstructive pulmonary disease. Am J Respir Crit Care Med. 2017;195 (3):324-330. doi:10.1164/rccm.201605-1014OC

6. Donaldson GC, Law M, Kowlessar B, et al. Impact of prolonged exacerbation recovery in chronic obstructive pulmonary disease. $\mathrm{Am}$ $J$ Respir Crit Care Med. 2015;192(8):943-950. doi:10.1164/ rccm.201412-2269OC

7. Mullerova H, Maselli DJ, Locantore N, et al. Hospitalized exacerbations of COPD: risk factors and outcomes in the ECLIPSE cohort. Chest. 2015;147(4):999-1007. doi:10.1378/chest.14-0655

8. Le Rouzic O, Roche N, Cortot AB, et al. Defining the "frequent exacerbator" phenotype in COPD: a hypothesis-free approach. Chest. 2018;153(5):1106-1115. doi:10.1016/j.chest.2017.10.009

9. Hurst JR, Vestbo J, Anzueto A, et al. Susceptibility to exacerbation in chronic obstructive pulmonary disease. N Engl J Med. 2010;363 (12):1128-1138. doi:10.1056/NEJMoa0909883

10. McGarvey L, Lee AJ, Roberts J, Gruffydd-Jones K, McKnight E, Haughney J. Characterisation of the frequent exacerbator phenotype in COPD patients in a large UK primary care population. Respir Med. 2015;109(2):228-237. doi:10.1016/j.rmed.2014.12.006

11. Nemeth T, Sperandio M, Mocsai A. Neutrophils as emerging therapeutic targets. Nat Rev Drug Discov. 2020;19(4):253-275.

12. Bantug GR, Galluzzi L, Kroemer G, Hess C. The spectrum of T cell metabolism in health and disease. Nat Rev Immunol. 2018;18 (1):19-34. doi:10.1038/nri.2017.99

13. Paliogiannis P, Fois AG, Sotgia S, et al. Neutrophil to lymphocyte ratio and clinical outcomes in COPD: recent evidence and future perspectives. Eur Respir Rev. 2018;27:147. doi:10.1183/ 16000617.0113-2017

14. Farah R, Ibrahim R, Nassar M, Najib D, Zivony Y, Eshel E. The neutrophil/lymphocyte ratio is a better addition to $\mathrm{C}$-reactive protein than CD64 index as a marker for infection in COPD. Panminerva Med. 2017;59(3):203-209. doi:10.23736/S0031-0808.17.03296-7

15. Brusselle GG, Joos GF, Bracke KR. New insights into the immunology of chronic obstructive pulmonary disease. Lancet. 2011;378 (9795):1015-1026. doi:10.1016/S0140-6736(11)60988-4 
16. Rovina N, Koutsoukou A, Koulouris NG. Inflammation and immune response in COPD: where do we stand? Mediators Inflamm. 2013;2013:413735. doi:10.1155/2013/413735

17. Lee H, Um SJ, Kim YS, et al. Association of the Neutrophil-toLymphocyte ratio with lung function and exacerbations in patients with chronic obstructive pulmonary disease. PLoS One. 2016;11(6): e0156511. doi:10.1371/journal.pone.0156511

18. Sorensen AK, Holmgaard DB, Mygind LH, Johansen J, Pedersen C. Neutrophil-to-lymphocyte ratio, calprotectin and YKL-40 in patients with chronic obstructive pulmonary disease: correlations and 5-year mortality - a cohort study. J Inflamm. 2015;12:20. doi:10.1186/ s12950-015-0064-5

19. Guan WJ, Liang WH, Zhao Y, et al. Comorbidity and its impact on 1590 patients with COVID-19 in China: a nationwide analysis. Eur Respir J. 2020;55:5. doi:10.1183/13993003.00547-2020

20. Zhang P, Zhu L, Cai J, et al. Association of inpatient use of angiotensin-converting enzyme inhibitors and angiotensin II receptor blockers with mortality among patients with hypertension hospitalized with COVID-19. Circ Res. 2020;126(12):1671-1681. doi:10.1161/CIRCRESAHA.120.317134

21. Miravitlles M, Soler-Cataluna JJ, Calle M, Soriano JB. Treatment of COPD by clinical phenotypes: putting old evidence into clinical practice. Eur Respir J. 2013;41(6):1252-1256. doi:10.1183/ 09031936.00118912

22. Lipson DA, Barnhart F, Brealey N, et al. Once-daily single-inhaler triple versus dual therapy in patients with COPD. $N$ Engl J Med. 2018;378(18):1671-1680. doi:10.1056/NEJMoa1713901

23. Matkovic Z, Huerta A, Soler N, et al. Predictors of adverse outcome in patients hospitalised for exacerbation of chronic obstructive pulmonary disease. Respiration. 2012;84(1):17-26. doi:10.1159/000335467

24. Barnes PJ. Inflammatory endotypes in COPD. Allergy. 2019;74 (7):1249-1256. doi:10.1111/all.13760

25. Singanayagam A, Loo SL, Calderazzo M, et al. Antiviral immunity is impaired in COPD patients with frequent exacerbations. Am J Physiol Lung Cell Mol Physiol. 2019;317(6):L893-L903. doi:10.1152/ ajplung.00253.2019
26. Geerdink JX, Simons SO, Pike R, Stauss HJ, Heijdra YF, Hurst JR. Differences in systemic adaptive immunity contribute to the 'frequent exacerbator' COPD phenotype. Respir Res. 2016;17(1):140. doi:10.1186/s12931-016-0456-y

27. Xiong W, Xu M, Zhao Y, Wu X, Pudasaini B, Liu JM. Can we predict the prognosis of COPD with a routine blood test? Int J Chron Obstruct Pulmon Dis. 2017;12:615-625. doi:10.2147/ COPD.S124041

28. Yao C, Liu X, Tang Z. Prognostic role of neutrophil-lymphocyte ratio and platelet-lymphocyte ratio for hospital mortality in patients with AECOPD. Int J Chron Obstruct Pulmon Dis. 2017;12:2285-2290. doi:10.2147/COPD.S141760

29. Teng F, Ye H, Xue T. Predictive value of neutrophil to lymphocyte ratio in patients with acute exacerbation of chronic obstructive pulmonary disease. PLoS One. 2018;13(9):e0204377. doi:10.1371/journal.pone.0204377

30. Salturk C, Karakurt Z, Adiguzel N, et al. Does eosinophilic COPD exacerbation have a better patient outcome than non-eosinophilic in the intensive care unit? Int J Chron Obstruct Pulmon Dis. 2015;10:1837-1846. doi:10.2147/COPD.S88058

31. Yun JH, Lamb A, Chase R, et al. Blood eosinophil count thresholds and exacerbations in patients with chronic obstructive pulmonary disease. J Allergy Clin Immunol. 2018;141(6):2037-2047 e2010. doi:10.1016/j.jaci.2018.04.010

32. Hastie AT, Martinez FJ, Curtis JL, et al. Association of sputum and blood eosinophil concentrations with clinical measures of COPD severity: an analysis of the SPIROMICS cohort. Lancet Respir Med. 2017;5(12):956-967. doi:10.1016/S2213-2600(17)30432-0

33. Wu HX, Zhuo KQ, Cheng DY. Peripheral blood eosinophil as a biomarker in outcomes of acute exacerbation of chronic obstructive pulmonary disease. Int $J$ Chron Obstruct Pulmon Dis. 2019;14:3003-3015. doi:10.2147/COPD.S226783

\section{Publish your work in this journal}

The International Journal of COPD is an international, peer-reviewed journal of therapeutics and pharmacology focusing on concise rapid reporting of clinical studies and reviews in COPD. Special focus is given to the pathophysiological processes underlying the disease, intervention programs, patient focused education, and self managemen protocols. This journal is indexed on PubMed Central, MedLine and CAS. The manuscript management system is completely online and includes a very quick and fair peer-review system, which is all easy to use. Visit http://www.dovepress.com/testimonials.php to read real quotes from published authors 